OPEN ACCESS

Edited by:

Arjan Te Pas,

Leiden University, Netherlands

Reviewed by:

Yi Shiau Ng,

Wellcome Trust Centre for Mitochondrial Research (WT),

United Kingdom

Joon Won Kang,

Chungnam National University,

South Korea

*Correspondence:

Hoon Park

hoondeng@yuhs.ac

tThese authors have contributed equally to this work

Specialty section:

This article was submitted to Pediatric Orthopedics, a section of the journa

Frontiers in Pediatrics

Received: 03 December 2020

Accepted: 14 October 2021

Published: 04 November 2021

Citation:

Kim S, Lee Y-M, Park K-B, Lee $M$ and Park H (2021) Point Prevalence and

Associated Factors of Hip

Displacement in Pediatric Patients

With Mitochondrial Disease.

Front. Pediatr. 9:637240

doi: 10.3389/fped.2021.637240

\section{Point Prevalence and Associated Factors of Hip Displacement in Pediatric Patients With Mitochondrial Disease}

\author{
Sungmin Kim ${ }^{1+}$, Young-Mock Lee ${ }^{2 \dagger}$, Kun-Bo Park ${ }^{3}$, Minsu Lee ${ }^{1}$ and Hoon Park ${ }^{4 *}$ \\ 1 Department of Orthopedic Surgery, Chonnam National University Medical School and Hospital, Gwangju, South Korea, \\ 2 Department of Pediatrics, Gangnam Severance Hospital, Yonsei University College of Medicine, Seoul, South Korea, \\ ${ }^{3}$ Division of Pediatric Orthopaedic Surgery, Severance Children's Hospital, Yonsei University College of Medicine, Seoul, \\ South Korea, ${ }^{4}$ Department of Orthopaedic Surgery, Gangnam Severance Hospital, Yonsei University College of Medicine, \\ Seoul, South Korea
}

Objective: Mitochondrial disease is a multisystem disorder resulting from mitochondrial dysfunction. Although musculoskeletal system is vulnerable to mitochondrial dysfunction, little information is available on orthopedic issues such as hip displacement and scoliosis in patients with mitochondrial disease. We aimed to examine the point prevalence of hip displacement and investigate the associated factors in patients with mitochondrial disease.

Methods: We retrospectively reviewed the medical records and plain radiographs of patients diagnosed with mitochondrial disease between January 2006 and January 2019 at a single institution. Data, including patient age, sex, follow-up duration, syndromic diagnosis, and gross motor function were collected. Migration percentage was measured on the radiographs. The clinical and radiologic variables were compared between patients classified according to the presence of hip displacement and motor function level.

Results: We included 225 patients (135 men, 90 women). The mean age at the latest follow-up was 11.1 years, and the mean follow-up duration was 7.0 years. Hip displacement was noted in $70(31.1 \%)$ patients. The proportion of patients with Leigh disease $(p=0.007)$ and the ratio of non-ambulators $(p<0.001)$ were higher among patients with hip displacement. The proportion of patients with Leigh disease was higher in the non-ambulators than the ambulators.

Conclusion: One-third of patients with mitochondrial disease developed hip displacement. Hip displacement was more common in non-ambulators or patients with hypertonia. Careful and serial monitoring for hip problems is required for non-ambulatory patients with mitochondrial disease who have increased muscle tone.

Keywords: mitochondrial disease, mitochondrial myopathy, Leigh syndrome, MELAS syndrome, hip displacement 


\section{INTRODUCTION}

Mitochondrial disease is a complex and heterogenous multisystem disorder resulting from mitochondrial dysfunction and, particularly, from defects of the mitochondrial respiratory chain (MRC) and associated abnormal oxidative phosphorylation (1). Different phenotypes and genotypes of mitochondrial disease may be noted in patients with abnormality in mitochondrial DNA or nuclear DNA, with variations in the extent and severity of the manifestations owing to the defects in any of the numerous mitochondrial pathways $(2,3)$. Clinical symptoms may develop at any age and may affect any organ (1).

The musculoskeletal system consists of organs with highenergy requirements and is vulnerable to mitochondrial dysfunction (1). Orthopedic manifestations are noted in patients with mitochondrial disease from a variety of causes, including underlying myopathy, neuropathy, and abnormalities of tone (4). Musculoskeletal involvement is noted in progressive proximal myopathy, exercise intolerance, muscle weakness, and gait disturbance $(3,5,6)$. Some patients with mitochondrial disease show severe musculoskeletal features, such as muscular contractures, scoliosis, hip dislocation, and limb deformities which may require surgical intervention. Only a few case reports on neuromuscular scoliosis in patients with mitochondrial disease have been published to date $(7,8)$.

Hip displacement can result in pain and difficulty during sitting and perineal care, leading to a considerable negative impact on the quality of life (9). Although many studies have reported on hip displacement in patients with neuromuscular diseases, like cerebral palsy, muscular dystrophy, or spinal muscular atrophy (9-11), to the best our knowledge, the occurrence and related factors of hip displacement in patients with mitochondrial disease have not been reported previously. This study aimed to examine the frequency of hip displacement in patients with mitochondrial disease and investigate the factors associated with the occurrence of hip displacement in these patients.

\section{MATERIALS AND METHODS}

We reviewed the medical records of patients who were diagnosed with mitochondrial disease and regularly followed up at our hospital between January 2006 and January 2019 at a single institution. Mitochondrial disease was diagnosed according to the modified criteria for mitochondrial disease proposed by Bernier et al. (12), which include clinical, histopathologic, enzymatic, and metabolic parameters. The inclusion criteria for the present study were as follows: availability of plain radiographs of the hip and spine for the evaluation and proper clinical records for assessing the medical and ambulatory status.

Data, including patient age at the initial and latest follow-up, sex, follow-up duration, gross motor function, and muscle tone were collected. The age of the patients when hip displacement occurred was estimated using serial radiographs. Gross motor function was assessed according to the walking status. Patients were divided into three groups-independent ambulators, ambulators with an assistive device, and non-ambulators. Muscle tone was classified as hypertonia, hypotonia, and normal. We investigated whether these patients underwent surgery. The type of surgery and postoperative complications were noted.

All patients were classified according to the syndromic diagnosis. The diagnoses of mitochondrial encephalomyopathy, lactic acidosis, and stroke-like episodes (MELAS) and Leigh disease were confirmed based on the diagnostic criteria reported by Yatsuga et al. (3) and Rahman et al. (13), respectively. Patients with non-specific and non-categorized mitochondrial disease did not show typical clinical symptoms, abnormal biochemical results, or genetic mutations conforming to the known and established mitochondrial syndromes (14). Genetic analysis and spectrophotometric biochemical enzyme assay for MRC complex of the muscle were performed for all patients.

Anteroposterior pelvic radiographs, taken with the hips slightly internally rotated, were used for radiologic measurements. All measurements were performed by two orthopedic surgeons. The migration percentage (15) and pelvic obliquity (16) were measured on pelvic radiographs at the initial and last follow-up. Hip subluxation requiring reconstructive surgery was defined according to the current literature as migration percentage $>30 \%$, and hip dislocation was defined as migration percentage of $100 \%(17,18)$. The degree of pelvic obliquity, the angle made by the horizontal line and line between the lowest points of the pelvic bones on the right and left sides, was also checked. The Cobb angle was measured from anteroposterior whole-spine radiographs. Scoliosis was defined as Cobb angle $>10^{\circ}$.

All data were analyzed using SPSS version 23 software (IBM Corp., Armonk, NY, USA). To compare the variables between groups with and without hip displacement, the two-sample $t$-test or Mann-Whitney $U$-test was used for continuous variables, and the $\chi^{2}$-test or Fisher's exact test was used for categorical variables. $p$-value $<0.05$ was considered significant for all analyses.

\section{RESULTS}

We identified 258 patients with mitochondrial disease. Thirtythree patients were excluded owing to inadequate radiographs for hip evaluation. The remaining 225 patients (135 men and 90 women) were included in this study. According to the syndromic diagnosis, 81 patients had Leigh syndrome, 10 had MELAS, and 134 had non-specific mitochondrial disease. Among 81 patients who met the clinical criteria for Leigh syndrome, 16 patients had confirmed the mitochondrial DNA mutation. Two patients showed nuclear DNA mutation and had SURF1 gene mutation. Ten patients diagnosed with MELAS were all positive mitochondrial DNA 3243A $>\mathrm{G}$ mutation. The majority of patients were not genetically confirmed in this study. The mean age at the latest follow-up was $11.1 \pm 5.3$ years, and the mean age at the initial visit was $4.8 \pm 3.9$ years. The mean follow-up duration was $7.0 \pm 3.9$ years (range, $1-24$ years).

Hip displacement was noted in $70(31.1 \%)$ patients. The estimated mean age when hip displacement occurred was 7.9 (3.2-12.7) years. The proportion of patients with hip subluxation and hip dislocation was 18.7 and $12.4 \%$, respectively (Figure 1A). 


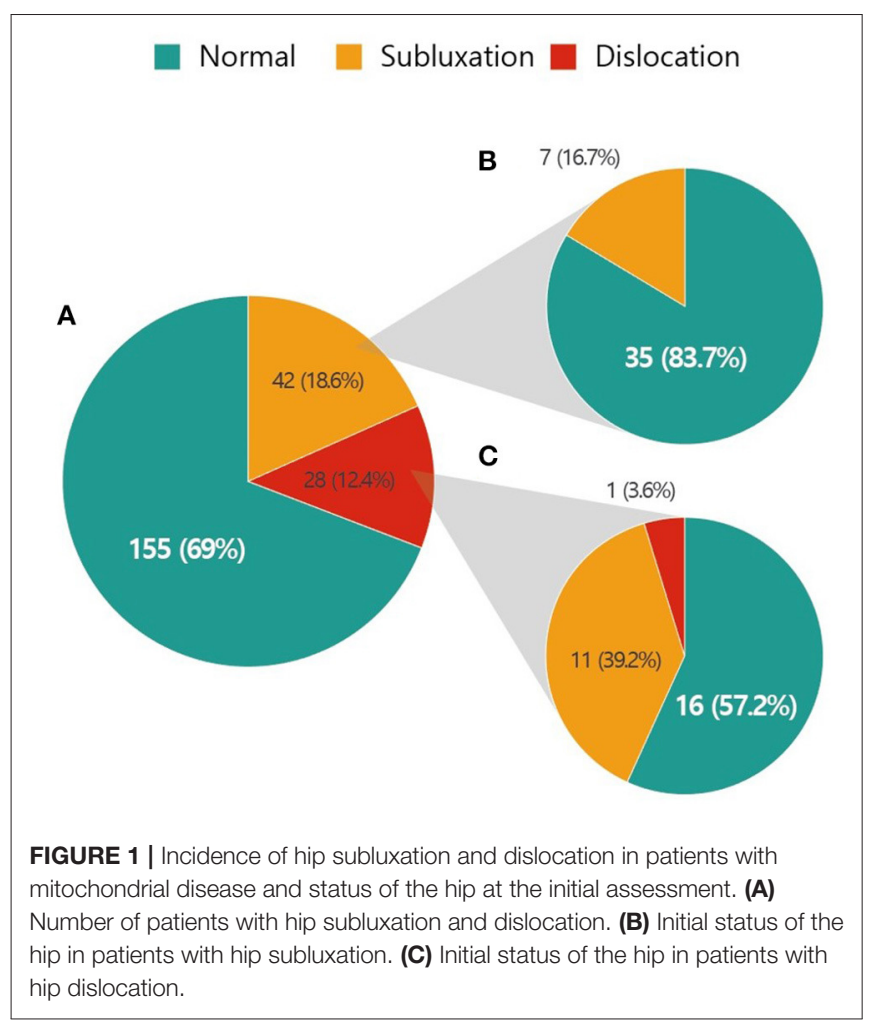

The status of the hip at the initial radiographic examination in patients with hip subluxation and dislocation is illustrated in Figures 1B,C, respectively. At the first assessment, 35 (83.3\%) of the 42 patients with hip subluxation were normal and $16(57.1 \%)$ of the 28 patients with hip dislocation were normal. According to syndromic diagnosis, the frequency of hip displacement was $43.2 \%$ (35 of 81 ) in patients with Leigh syndrome, $10 \%$ ( 1 of 10 ) in patients with MELAS, and $25.4 \%$ (34 of 134) in patients with non-specific and non-categorized mitochondrial disease.

Sixty-six (29.3\%) patients were independent ambulators, 37 (16.4\%) were able to ambulate with an assistive device, and 122 (54.2\%) were non-ambulators. The distribution of hip displacement according to motor function level is presented in Figure 2. The occurrence of hip displacement was found in 9 (8.7\%) patients who were able to walk and in 61 (50\%) nonambulatory patients. The frequency of hip subluxation was $4.5 \%$ in independent ambulators, $16.2 \%$ in ambulators with an assistive device, and $25.4 \%$ in non-ambulators. While hip dislocation was not found in ambulatory patients, it was noted in 30 (24.6\%) non-ambulatory patients.

Seventy-five $(33.3 \%)$ patients had increased muscle tone, 29 (12.9\%) patients had decreased muscle tone, and 72 (32\%) patients showed normal muscle tone. The muscle tone in 49 (21.8\%) patients cannot be evaluated because of the lack of medical record. Hypertonia was found in 39 (48.1\%) patients with Leigh syndrome, and 35 (26.1\%) patients with non-specific mitochondrial disease.

Mitochondrial respiratory chain complex deficiency was found in $191(84.8 \%)$ patients, and the results showed complex
I deficiency in 165 cases (86.4\%), complex II deficiency in one case $(0.5 \%)$, and complex IV deficiency in 25 cases $(13.1 \%)$.

Forty-three $(19.1 \%)$ patients had scoliosis, and the mean Cobb's angle was $48.2^{\circ}$. Hip displacement was noted in 16 patients with scoliosis. Pelvic obliquity was present in $7.1 \%$, and the mean pelvic obliquity was $19.3^{\circ}$.

Comparisons of the characteristics between patients with and without hip displacement are described in Table 1. The proportion of patients with Leigh disease was higher among patients with hip displacement than among those without $(p=$ $0.007)$. Non-ambulators were more commonly noted among the group with hip displacement $(p<0.001)$. There was a significant difference in the mean follow-up period between the two groups. Significant differences in age, sex, MRC deficiency, and presence of scoliosis and pelvic obliquity were not found between the two groups.

We also compared the variables between ambulators and nonambulators (Table 2). Age $(p=0.012)$, distribution of syndromic diagnosis $(p=0.027)$, and presence of scoliosis $(p=0.027)$ significantly differed between the two groups. The proportions of patients with Leigh disease and scoliosis were higher among the non-ambulators.

Ten patients underwent surgical treatment for hip displacement. Among five patients with Leigh syndrome, three patients underwent hip reconstruction surgery (femur and pelvic osteotomy) while other two underwent soft tissue release. Hip reconstruction surgery was done in five patients with non-specific mitochondrial disease. There was no redislocation of hip in patients managed with surgery during the study period. Three patients received the posterior spinal fusion for scoliosis. Bracing was used in 10 patients, and wheelchair modification, physical therapy, and environmental adaptation were used in the remaining 30 patients. All patients who underwent surgery had increased muscle tone.

\section{DISCUSSION}

This is the first report to describe the occurrence of hip displacement in patients with mitochondrial disease. Hip displacement developed in $31.1 \%$ of patients with mitochondrial disease in our cohort. The point prevalence of hip displacement in our study is similar to that reported in patients with cerebral palsy $(18,19)$. The true incidence of hip displacement in patients with mitochondrial disease is difficult to determine because of the rarity of mitochondrial disease. However, the number of patients in our cohort is large enough to be considered meaningful.

In our study, the status of the hip in most patients with hip displacement was normal at the initial visit. This may have been because of either late-onset hip displacement or the failure to detect hip displacement at the initial assessment. Although it is unclear whether hip displacement is present at birth or can develop over time, our results imply that hip displacement in patients with mitochondrial disease may progress with age. This result suggests that hip displacement should be regularly monitored in patients with mitochondrial disease. 


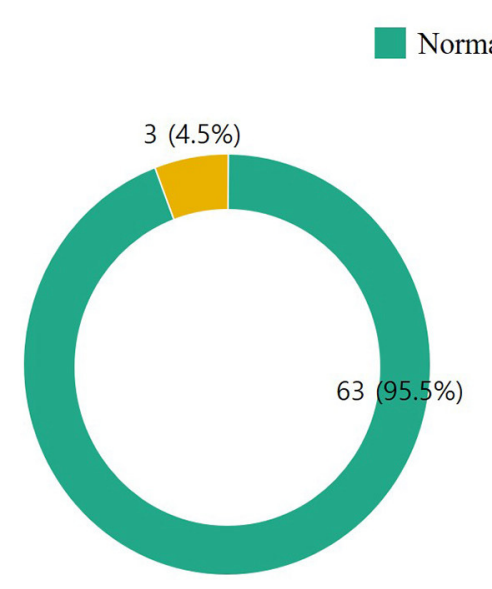

A Independent Ambulator

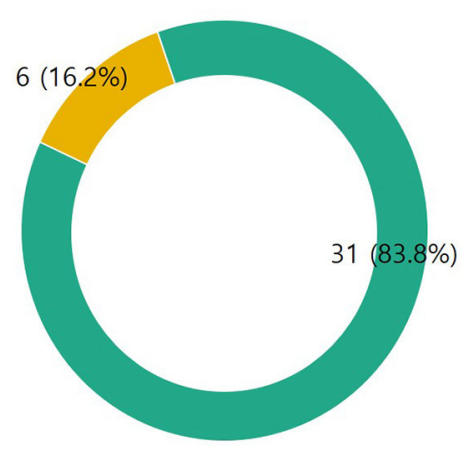

B Ambulator with assistive device

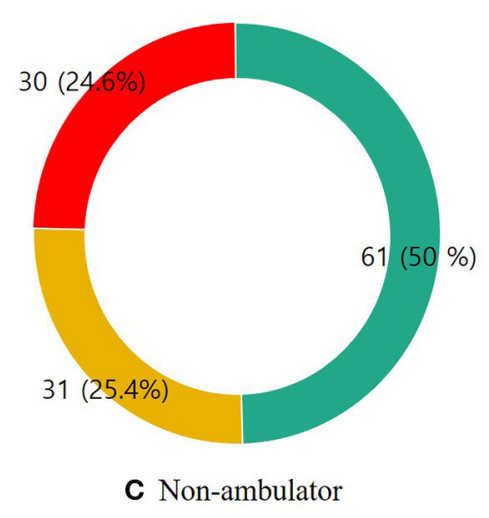

C Non-ambulator

FIGURE 2 | Hip displacement in patients with mitochondrial disease according to the motor function level. (A) Independent ambulatory, (B) ambulator with assistive device, and (C) non-ambulator.

Our results showed that hip displacement was strongly associated with the motor function level. The frequency of hip displacement was higher in non-ambulators. These results were similar to those of other studies that confirmed the linear association between Gross Motor Function Classification System (GMFCS) level and hip displacement in patients with cerebral palsy $(18,19)$. As the GMFCS is specific to cerebral palsy and cannot be directly applied to patients with mitochondrial disease, we classified the patients in this study into three groups according to motor function level, as follows: independent ambulators, ambulators with an assistive device, and nonambulators. Dislocation was not noted in ambulatory patients and was noted only in non-ambulatory patients. The present data showed that non-ambulatory patients with mitochondrial disease had a high risk for hip displacement or dislocation. Therefore, physicians must pay more attention to non-ambulatory patients with mitochondrial disease.

Our study reported that the proportion of patients with Leigh disease was higher in the group with hip displacement. Leigh syndrome is a neurodegenerative disease characterized by bilateral central nervous system lesions (14). Leigh syndrome is a heterogenous neurodegenerative condition that affects the basal ganglia, the thalamus, and the brainstem (20). Affected individuals may develop weak muscle tone (hypotonia), involuntary muscle contractions (dystonia), muscle spasm (spasticity), and problems with movement and balance (ataxia) (21). Although we could not identify the muscle tone in all included patients, the proportion of patients with hypertonia in Leigh syndrome was higher than that of non-specific mitochondrial disease. This result may support that increased muscle tone is the main factor causing the hip displacement in patients with Leigh syndrome. Although spasticity is an important cause of hypertonia, increased muscle tone may also be attributable to dystonia when there is simultaneous co-contraction of agonist and antagonist muscles at rest (22).
It is well-known that dystonia is one of common feature of mitochondrial disease such as Leigh syndrome (21). Muscle imbalance due to increased or spastic muscle tone induce abnormal force on the hip joint $(18,23)$. Over time, the relatively increased activity of the hip adductors and flexors abnormally alter the joint reactive force, gradually causing the proximal femur to subluxate from the acetabulum (17). These forces also create anatomic differences and prevent the physiologic remodeling of immature hip geometry including increased anteversion, increased neck-shaft angle, and acetabular dysplasia (24). The resulting pathology can lead to hip subluxation and eventual dislocation, which may deteriorate the motor function level. Although, muscular imbalance can also occur in patients with hypotonic or flaccid muscle tone, hip displacement is common in patients with increased or spastic muscle tone such as in cerebral palsy. We assume that hypertonia is the main factor causing hip displacement even in patients with mitochondrial disease.

The frequency of scoliosis in patients with mitochondrial disease was $19.1 \%$. Only two case reports on scoliosis in patients with mitochondrial disease have been published to date $(7,8)$. Although they reported a 5\% incidence of scoliosis among patients with mitochondrial myopathies, the total number of patients was only 60. Therefore, it is not reasonable to compare this rate with that in our study.

The distribution of scoliosis according to the presence of hip displacement was not different. Scoliosis is frequently observed in patients with neuromuscular disease (14, 25-27). Although hip displacement may accompany scoliosis, previous studies investigating the causal relationship between these factors showed inconsistent results (28-33). Further studies are required to evaluate the association between hip displacement and scoliosis in patients with neuromuscular diseases.

The proportion of patients with scoliosis was higher among the non-ambulators than among the ambulators. In patients 
TABLE 1 | Distribution of the variables according to hip displacement.

\begin{tabular}{|c|c|c|c|}
\hline & $\begin{array}{c}\text { Hip } \\
\text { displacement } \\
(n=70)\end{array}$ & $\begin{array}{c}\text { No hip } \\
\text { displacement } \\
(n=155)\end{array}$ & $p$-Value \\
\hline Age, mean $\pm S D$, years & $10.4 \pm 3.5$ & $11.6 \pm 6.4$ & 0.076 \\
\hline Sex, $n(\%)$ & & & 0.661 \\
\hline Male & $40(57.1)$ & $94(60.6)$ & \\
\hline Female & 30 (42.9) & 61 (39.4) & \\
\hline $\begin{array}{l}\text { Mean follow-up, mean } \pm \\
\text { SD, years }\end{array}$ & $8.4 \pm 3.3$ & $6.7 \pm 3.9$ & 0.021 \\
\hline Syndrome, $n(\%)$ & & & 0.007 \\
\hline Leigh & $35(50.0)$ & $46(29.7)$ & \\
\hline MELAS & $1(1.4)$ & $9(5.8)$ & \\
\hline NS\&NC MD & 34 (48.6) & $100(64.5)$ & \\
\hline Motor function level, $n$ (\%) & & & $<0.001$ \\
\hline \multicolumn{4}{|l|}{ Ambulatory } \\
\hline Without aids & $3(4.3)$ & $63(40.6)$ & \\
\hline With aids & $6(8.6)$ & $31(20.0)$ & \\
\hline Non-ambulatory & $61(87.1)$ & $61(39.4)$ & \\
\hline Muscle tone, $n(\%)$ & & & $<0.001$ \\
\hline Hypertonia & $42(60.0)$ & 33 (21.3) & \\
\hline Hypotonia & $12(17.1)$ & $17(11.0)$ & \\
\hline Normal tone & $5(7.1)$ & $67(43.2)$ & \\
\hline Unknown & $11(15.7)$ & $38(24.5)$ & \\
\hline MRC deficiency, $n(\%)$ & & & 0.174 \\
\hline Complex I deficiency & $47(67.1)$ & $118(76.1)$ & \\
\hline Complex II deficiency & $1(1.4)$ & $0(0)$ & \\
\hline Complex IV deficiency & $11(15.7)$ & $14(9.0)$ & \\
\hline $\begin{array}{l}\text { Absence of MRC } \\
\text { deficiency }\end{array}$ & $11(15.7)$ & $23(14.8)$ & \\
\hline Scoliosis, $n(\%)$ & & & 0.362 \\
\hline Yes & $16(22.9)$ & $27(17.4)$ & \\
\hline No & $54(77.1)$ & $128(82.6)$ & \\
\hline Pelvic obliquity, $n$ (\%) & & & 0.100 \\
\hline Yes & $8(11.4)$ & $8(5.2)$ & \\
\hline No & 62 (88.6) & $147(94.8)$ & \\
\hline
\end{tabular}

MELAS, mitochondrial encephalomyopathy, lactic acidosis, and stroke-like episodes; NS\&NC MD, non-specific and non-categorized mitochondrial disease; MRC, mitochondrial respiratoy chain complex.

with cerebral palsy, the risk of scoliosis was closely associated with the ambulation status (34). Low motor function level due to muscle weakness and incomplete muscle control contributes to impaired trunk control and the development of spinal deformity. Our results showed that ambulation status was related to preserve the development of the hip joint in patients with mitochondrial disease.

Although we found a high incidence of hip displacement in patients with mitochondrial disease, there are no specific treatment guidelines and surgical techniques for these patients. Some patients with mitochondrial disease have very unhealthy medical condition and show high mortality rates from sepsis or pneumonia (1). It may be unnecessary to evaluate and monitor the status of the hip joint in these patients. However,
TABLE 2 | Variables distribution according to ambulation.

\begin{tabular}{lccc}
\hline & $\begin{array}{c}\text { Ambulatory } \\
(\boldsymbol{n}=\mathbf{1 0 3})\end{array}$ & $\begin{array}{c}\text { Non-ambulatory } \\
(\boldsymbol{n}=\mathbf{1 2 2})\end{array}$ & $\boldsymbol{p}$-Value \\
\hline Age, mean \pm SD, years & $12.3 \pm 5.8$ & $10.4 \pm 5.4$ & 0.012 \\
Sex, $n$ (\%) & $66(64.1)$ & $68(55.7)$ & 0.222 \\
Male & $37(35.9)$ & $54(44.3)$ & \\
Female & & & 0.027 \\
Syndrome, $n$ (\%) & $28(27.2)$ & $53(43.4)$ & \\
Leigh & $4(3.9)$ & $6(4.9)$ & \\
MELAS & $71(68.9)$ & $63(51.7)$ & \\
NS\&NC MD & & & \\
Muscle tone, $n$ (\%) & $19(18.4)$ & $56(45.9)$ & \\
Hypertonia & $3(2.9)$ & $26(21.3)$ & \\
Hypotonia & $58(56.3)$ & $14(11.5)$ & \\
Normal tone & $23(22.3)$ & $26(21.3)$ & \\
Unknown & & & \\
Scoliosis, $n$ (\%) & $13(12.6)$ & $30(24.6)$ & \\
Yes & $90(87.4)$ & $92(75.4)$ & \\
No & & & \\
Pelvic obliquity, $n$ (\%) & $4(3.9)$ & $12(9.8)$ & \\
Yes & $99(96.1)$ & $110(90.2)$ & \\
No & & \\
\hline
\end{tabular}

MELAS, Mitochondrial encephalomyopathy, lactic acidosis, and stroke-like episodes; NS\&NC MD, non-specific and non-categorized mitochondrial disease.

we consider that serial evaluation for hip displacement is important because it may be cost-effective to preserve the hip joint by physical therapy or minor surgery, like soft tissue release (6). Considering the estimated mean age when hip displacement occurred, we recommend that hip radiography examination, such as the cerebral palsy surveillance program, ideally be initiated by $6-8$ years of age in nonambulatory patients with mitochondrial diseases. Surveillance frequency is based on the child's age, GMFCS level, and muscle tone.

This study had some limitations. First, our study is retrospective and cross-sectional in nature. We could not examine the same periods in all patients owing to the variable periods of follow-up, which is possibly why we found a significant difference in the mean follow-up periods between patients with and without hip displacement. This may be because nonambulators with hip displacement frequently visit the hospital for medical care or checkups for the hip joint status, whereas ambulators without hip displacement do not need additional radiography examination. Additionally, some patients had not reached skeletal maturity at the final assessment, which may have led to a bias in assessing the incidence of hip displacement. As noted earlier, this is the first report on hip displacement in a large patient group with mitochondrial disease. Second, we included a smaller proportion of patients with MELAS because it is an extremely rare disease subtype. Thus, the frequency of hip displacement in patients with MELAS must be interpreted with caution. 
In conclusion, $31.1 \%$ of patients with mitochondrial disease developed hip displacement. Hip displacement was more common in non-ambulators or patients with increased muscle tone. Careful and serial monitoring for hip problems are recommended in non-ambulatory patients with mitochondrial disease who have increased muscle tone.

\section{DATA AVAILABILITY STATEMENT}

The original contributions presented in the study are included in the article/supplementary material, further inquiries can be directed to the corresponding author/s.

\section{ETHICS STATEMENT}

The studies involving human participants were reviewed and approved by the Institutional Review Board of the Gangnam Severance Hospital, Seoul, Korea. Written informed consent for participation was not provided by the participants' legal

\section{REFERENCES}

1. Eom S, Lee HN, Lee S, Kang HC, Lee JS, Kim HD, et al. Cause of death in children with mitochondrial diseases. Pediatr Neurol. (2017) 66:82-8. doi: 10.1016/j.pediatrneurol.2016.10.006

2. Eom S, Lee Y-M. Preliminary study of neurodevelopmental outcomes and parenting stress in pediatric mitochondrial disease Pediatr Neurol. (2017) 71:43.e1-9.e1. doi: 10.1016/j.pediatrneurol.2017.01.019

3. Yatsuga S, Povalko N, Nishioka J, Katayama K, Kakimoto N, Matsuishi T, et al. MELAS: a nationwide prospective cohort study of 96 patients in Japan. Biochim Biophys Acta. (2012) 1820:619-24. doi: 10.1016/j.bbagen.2011.03.015

4. Parikh S, Goldstein A, Karaa A, Koenig MK, Anselm I, Brunel-Guitton C, et al. Patient care standards for primary mitochondrial disease: a consensus statement from the Mitochondrial Medicine Society. Genet Med. (2017) 19:10.1038/gim.2017.107. doi: 10.1038/gim.2017.107

5. McFarland R, Taylor RW, Turnbull DM. The neurology of mitochondrial DNA disease. Lancet Neurol. (2002) 1:343-51. doi: 10.1016/S1474-4422(02)00159-X

6. Kisler JE, Whittaker RG, McFarland R. Mitochondrial diseases in childhood: a clinical approach to investigation and management. Dev Med Child Neurol. (2010) 52:422-33. doi: 10.1111/j.1469-8749.2009.03605.x

7. Li Z, Shen J, Liang J. Scoliosis in mitochondrial myopathy: case report and review of the literature. Medicine (Baltimore). (2015) 94:e513. doi: 10.1097/MD.0000000000000513

8. Hiniker A, Wong LJ, Berven S, Truong CK, Adesina AM, Margeta M. Axial mitochondrial myopathy in a patient with rapidly progressive adult-onset scoliosis. Acta Neuropathol Commun. (2014) 2:137. doi: 10.1186/s40478-014-0137-3

9. Huser A, Mo M, Hosseinzadeh P. Hip surveillance in children with cerebral palsy. Orthop Clin North Am. (2018) 49:181-90. doi: 10.1016/j.ocl.2017.11.006

10. Chan KG, Galasko CS, Delaney C. Hip subluxation and dislocation in Duchenne muscular dystrophy. J Pediatr Orthop B. (2001) 10:219-25. doi: 10.1097/01202412-200107000-00012

11. Sporer SM, Smith BG. Hip dislocation in patients with spinal muscular atrophy. J Pediatr Orthop. (2003) 23:10-4. doi: 10.1097/01241398-200301000-00002

12. Bernier FP, Boneh A, Dennett X, Chow CW, Cleary MA, Thorburn DR. Diagnostic criteria for respiratory chain disorders in adults and children. Neurology. (2002) 59:1406-11. doi: 10.1212/01.WNL.0000033795.17156.00

13. Rahman S, Blok RB, Dahl HH, Danks DM, Kirby DM, Chow CW, et al. Leigh syndrome: clinical features and biochemical and DNA abnormalities. Ann Neurol. (1996) 39:343-51. doi: 10.1002/ana.410390311 guardians/next of kin because: Our research involved no more than a minimal risk to our subjects and we used the existing medical records and the radiographs.

\section{AUTHOR CONTRIBUTIONS}

The initial concept was prepared by Y-ML and HP. The initial draft was prepared by SK. Data was collected by ML and SK. Data analysis was performed by SK and K-BP. The final draft was prepared by SK, Y-ML, and HP. Intellectual contents were provided by K-BP and HP. All authors read the final manuscript and approved it for submission.

\section{FUNDING}

This research was funded by a faculty research grant of Yonsei University College of Medicine, grant number 6-2020-0123. The APC was funded by Yonsei University College of Medicine.

14. Carroll NC. Assessment and management of the lower extremity in myelodysplasia. Orthop Clin North Am. (1987) 18:709-24. doi: 10.1016/S0030-5898(20)30360-6

15. Reimers J. The stability of the hip in children: a radiological study of the results of muscle surgery in cerebral palsy. Acta Orthop Scand Suppl. (1980) 184:1-100. doi: 10.3109/ort.1980.51.suppl-184.01

16. Hagglund G, Goldring M, Hermanson M, Rodby-Bousquet E. Pelvic obliquity and measurement of hip displacement in children with cerebral palsy. Acta Orthop. (2018) 89:652-5. doi: 10.1080/17453674.2018. 1519104

17. Shore BJ, Graham HK Jr. Management of moderate to severe hip displacement in nonambulatory children with cerebral palsy. JBJS Rev. (2017) 5:e4. doi: 10.2106/JBJS.RVW.17.00027

18. Soo B, Howard JJ, Boyd RN, Reid SM, Lanigan A, Wolfe R, et al. Hip displacement in cerebral palsy. J Bone Joint Surg Am. (2006) 88:121-9. doi: 10.2106/00004623-200601000-00015

19. Connelly A, Flett P, Graham HK, Oates J. Hip surveillance in Tasmanian children with cerebral palsy. J Paediatr Child Health. (2009) 45:437-43. doi: 10.1111/j.1440-1754.2009.01534.x

20. Horvath R, Abicht A, Holinski-Feder E, Laner A, Gempel K, Prokisch H, et al. Leigh syndrome caused by mutations in the flavoprotein (FP) subunit of succinate dehydrogenase (SDHA). J Neurol Neurosurg Psychiatry. (2006) 77:74-6. doi: 10.1136/jnnp.2005.067041

21. Ghaoui R, Sue CM. Movement disorders in mitochondrial disease. J Neurol. (2018) 265:1230-40. doi: 10.1007/s00415-017-8722-6

22. Sanger TD. Pathophysiology of pediatric movement disorders. J Child Neurol. (2003) 18(Suppl 1):S9-24. doi: 10.1177/0883073803018001S0401

23. Hagglund G, Lauge-Pedersen H, Wagner P. Characteristics of children with hip displacement in cerebral palsy. BMC Musculoskelet Disord. (2007) 8:101. doi: 10.1186/1471-2474-8-101

24. Shrader MW, Wimberly L, Thompson R. Hip surveillance in children with cerebral palsy. J Am Acad Orthop Surg. (2019) 27:760-8. doi: 10.5435/JAAOS-D-18-00184

25. Shapiro F, Zurakowski D, Bui T, Darras BT. Progression of spinal deformity in wheelchair-dependent patients with Duchenne muscular dystrophy who are not treated with steroids: coronal plane (scoliosis) and sagittal plane (kyphosis, lordosis) deformity. Bone Joint J. (2014) 96:100-5. doi: 10.1302/0301-620X.96B1.32117

26. Garg S, Engelman G, Yoshihara H, McNair B, Chang F. The relationship of gross motor functional classification scale level and hip dysplasia on the pattern and progression of scoliosis in children with cerebral palsy. Spine Deform. (2013) 1:266-71. doi: 10.1016/j.jspd.2013.05.002 
27. Fujak A, Raab W, Schuh A, Richter S, Forst R, Forst J. Natural course of scoliosis in proximal spinal muscular atrophy type II and IIIa: descriptive clinical study with retrospective data collection of 126 patients. BMC Musculoskelet Disord. (2013) 14:283. doi: 10.1186/1471-2474-14-283

28. Senaran H, Shah SA, Glutting JJ, Dabney KW, Miller F. The associated effects of untreated unilateral hip dislocation in cerebral palsy scoliosis. J Pediatr Orthop. (2006) 26:769-72. doi: 10.1097/01.bpo.0000242426. 60995.29

29. Lonstein JE, Beck K. Hip dislocation and subluxation in cerebral palsy. $J$ Pediatr Orthop. (1986) 6:521-6. doi: 10.1097/01241398-198609000-00001

30. Pritchett JW. The untreated unstable hip in severe cerebral palsy. Clin Orthop Relat Res. (1983) 173:169-72. doi: 10.1097/00003086-198303000-00022

31. Patel J, Shapiro F. Simultaneous progression patterns of scoliosis, pelvic obliquity, and hip subluxation/dislocation in non-ambulatory neuromuscular patients: an approach to deformity documentation. J Child Orthop. (2015) 9:345-56. doi: 10.1007/s11832-015-0683-7

32. Repko M, Krbec M, Chaloupka R, Tichý V, Sprláková-Puková A. Neuromuscular deformity of the pelvis and its surgical treatment. Acta Chir Orthop Traumatol Cech. (2008) 75:117.

33. Frischhut B, Krismer M, Stoeckl B, Landauer F, Auckenthaler T. Pelvic tilt in neuromuscular disorders. J Pediatr Orthop B. (2000) 9:221-8. doi: 10.1097/01202412-200010000-00003
34. Hägglund G, Pettersson K, Czuba T, Persson-Bunke M, RodbyBousquet E. Incidence of scoliosis in cerebral palsy: a population-based study of 962 young individuals. Acta Orthop. (2018) 89:443-7. doi: $10.1080 / 17453674.2018 .1450091$

Conflict of Interest: The authors declare that the research was conducted in the absence of any commercial or financial relationships that could be construed as a potential conflict of interest.

Publisher's Note: All claims expressed in this article are solely those of the authors and do not necessarily represent those of their affiliated organizations, or those of the publisher, the editors and the reviewers. Any product that may be evaluated in this article, or claim that may be made by its manufacturer, is not guaranteed or endorsed by the publisher.

Copyright (c) 2021 Kim, Lee, Park, Lee and Park. This is an open-access article distributed under the terms of the Creative Commons Attribution License (CC BY). The use, distribution or reproduction in other forums is permitted, provided the original author(s) and the copyright owner(s) are credited and that the original publication in this journal is cited, in accordance with accepted academic practice. No use, distribution or reproduction is permitted which does not comply with these terms. 